\title{
Inclusive Jet Production at CDF
}

\author{
Christina Mesropian ${ }^{1}$, on behalf of the CDF Collaboration \\ ${ }^{1}$ The Rockefeller University \\ 1230 York Avenue \\ New York, NY 10021, USA
}

Received on 21 October, 2006; revised version received on 23 March, 2007

\begin{abstract}
We present results on inclusive jet production in proton-antiproton collisions at $\sqrt{s}=1.96 \mathrm{TeV}$. The measurements are based on $1.0 \mathrm{fb}^{-1}$ of CDF Run II data and were carried out for jets in five different jet rapidity regions up to $|Y|=2.1$. Both the midpoint cone based algorithm and $k_{T}$ algorithm were investigated to reconstruct the jets. The results are compared to next-to-leading order perturbative QCD predictions.
\end{abstract}

Keywords: QCD; Inclusive jet production

\section{INTRODUCTION}

The measurement of the inclusive jet cross section represents one of the basic tests of QCD at hadron-hadron colliders. As a function of jet transverse momentum, $p_{T}^{j e t}$, the cross section extends over eight orders of magnitude allowing to probe the distances as small as $10^{-19} \mathrm{~m}$.

The Tevatron Run I measurement of the inclusive jet cross section [1] which showed apparent excess at high transverse energy generated substantial interest in the particle physics community and motivated the reevaluation of the theoretical uncertainties from parton distribution functions (PDF's). Current PDF sets [2] now exploit the flexibility of gluon distributions at high $x$ values, which can account for the excess observed in the data at high $E_{T}$, and include Run I jet data in the global fits.

Increased center-of-mass energy (from 1.8 to $1.96 \mathrm{TeV}$ ) and high luminosity in Run II resulted in a dramatically extended kinematic range for jet cross section measurements, both in $p_{T}^{j e t}$ and jet rapidity, $Y$. Since the inclusive jet measurement in the forward region probes a kinematic region which is not expected to be sensitive to new physics, these results could become a powerful constraint on the gluon PDF, thus improving theoretical predictions in all physics channels for experiments at the Tevatron and LHC.

\section{JET RECONSTRUCTION}

The cornerstone of data to theory comparisons is the question of jet identification. In theoretical calculations, jets are manifestations of partons as relatively isolated sprays of energetic hadrons observed in the final state of high energy collisions. From the experimental point of view jets are defined as large energy deposits in a localized group of calorimeter cells, see Fig. 1. To minimize the difference between parton level predictions and measured jet properties, the jet algorithms, which could be implemented for both situations, are used. In Run II, CDF collaboration explored alternative jet algorithms as the cone algorithm used in Run I is not collinear and infrared safe. Jets are now reconstructed with two algorithms: MidPoint algorithm [3] and $k_{T}$ algorithm [4].
The MidPoint algorithm is an iterative seed-based cone algorithm that uses midpoints between a pair of protojets as additional seeds in order to make the clusterization procedure infrared safe. A cone with radius $R=0.7$ in $Y-\phi$ plane is used with an additional prescription to deal with overlapping cones and merges: two protojets are merged if the common transverse momentum is larger than $75 \%$ of that of the jet with the less transverse momentum, otherwise two jets are formed and the common towers are assigned to the closest jet. To regulate merging and separation of parton clusters in a manner similar to the experimental analysis, the theoretical NLO QCD calculation introduces an ad hoc parameter $R_{\text {sep }}$ to merge two partons if they are within $R_{\text {cone }} \times R_{\text {sep }}$ of each other and within $R_{\text {cone }}$ of the resulting jet centroid. Based on parton level approximate arguments $R_{\text {sep }}$ is set to 1.3 .

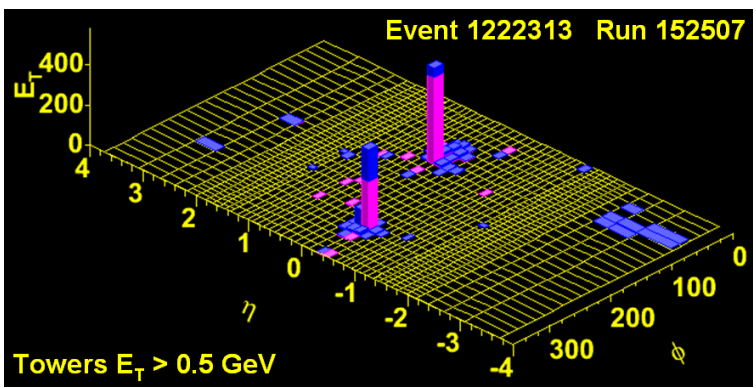

FIG. 1: Jet events in the CDF calorimeter.

The $k_{T}$ algorithm, inspired by pQCD gluon emission, is infrared and collinear safe to all orders in pQCD. The algorithm pairs nearby protojets in order of increasing relative transverse momentum and contains a parameter $D$, that controls the size of resulting jets. The advantage of $k_{T}$ algorithm relative to the cone-based jet algorithms is the absence of merging/splitting prescriptions which makes it preferable for comparisons with theory, however it is more sensitive to soft contributions, such as underlying event or multiple $p \bar{p}$ interactions per bunch crossing. Although $k_{T}$ algorithm was successfully used at HERA, its performance at hadron-hadron colliders was more challenging. 


\section{MEASUREMENT OF THE INCLUSIVE JET CROSS SECTION WITH MIDPOINT ALGORITHM}

In this contribution we report results of the inclusive jet cross section measurement with MidPoint jet finding algorithm based on $1.0 \mathrm{fb}^{-1}$ of CDF Run II data. The Fig. 2 shows the measured cross section for different rapidity regions as a function of jet transverse momentum, which extends to over $600 \mathrm{GeV} / \mathrm{c}$. The experimental systematic uncertainties, repre-



FIG. 2: The inclusive jet cross section with MidPoint algorithm in the different rapidity regions. The different rapidity region cross sections are scaled by a given factor for presentation purposes.

sented as the yellow band, are dominated by the uncertainty on the absolute jet energy scale, which is know at the level of $\pm 2 \%$ for low $p_{T}^{j e t}$ and $\pm 3 \%$ for high $p_{T}^{j e t}$ [5]. This translates

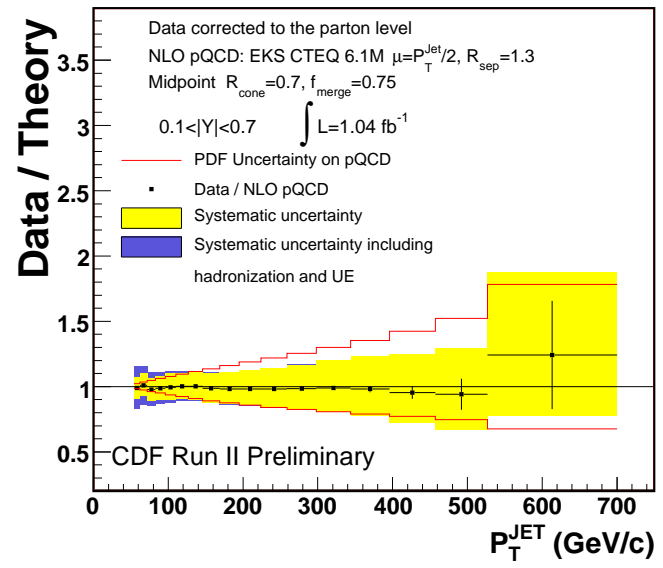

FIG. 3: The ratio of measured inclusive jet cross section corrected to the parton data to the $\mathrm{pQCD}$ predictions for central jets with rapidity $0.1<|Y|<0.7$.

to the uncertainty on the measured cross section from $20 \%$ to $40 \%$. An additional normalization uncertainty of $\pm 6 \%$ due to the luminosity measurement is not included in the Figure. The experimental results are compared with theoretical predictions obtained with NLO QCD program EKS [6] with CTEQ6.1M as an input PDF. The factorization and renormalization scales are being set to $p_{T}^{\text {jet }} / 2$.

To be able to have direct comparison to theory the corrections for non-perturbative contributions, coming from the underlying events and the hadronization processes, should be made. These corrections are obtained with PYTHIA 6.203 [7] as the ratio of the predicted inclusive cross sections at the hadron level and at the parton level (before fragmentation into hadrons and without multiple parton interactions). A special set of parameters, tuned on Run I CDF data to reproduce underlying event activity and denoted PYTHIA-Tune A [8] was used. The same corrections were being evaluated with HERWIG6.4 [9], with difference between two Monte Carlo simulations being considered as the systematic uncertainty of this correction.

Fig. 3 shows ratio between data and theory for central jets $(0.1<|Y|<0.7)$ with data already being corrected to the parton level. Good agreement with NLO pQCD predictions are found for entire $p_{T}^{\text {jet }}$ range. The blue band shows the systematic uncertainties with underlying event and hadronization uncertainties included, these contributions being important at low $p_{T}^{\text {jet }}$. The red line represents uncertainties of theoretical predictions which are dominated mostly by the choice of input PDFs, especially by the limited knowledge of the gluon distributions at high $x$.

\section{MEASUREMENT OF THE INCLUSIVE JET CROSS SECTION WITH $k_{T}$ ALGORITHM}

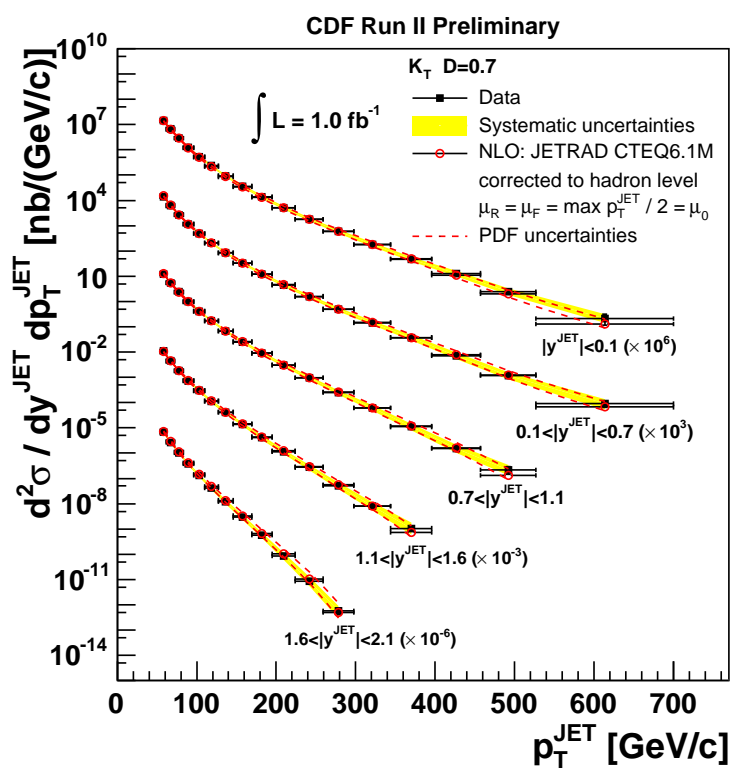

FIG. 4: The inclusive jet cross section with $k_{T}$ algorithm in the different rapidity regions.

Recently published CDF results [10] on the inclusive jet cross section with $k_{T}$ algorithm demonstrated that this algo- 


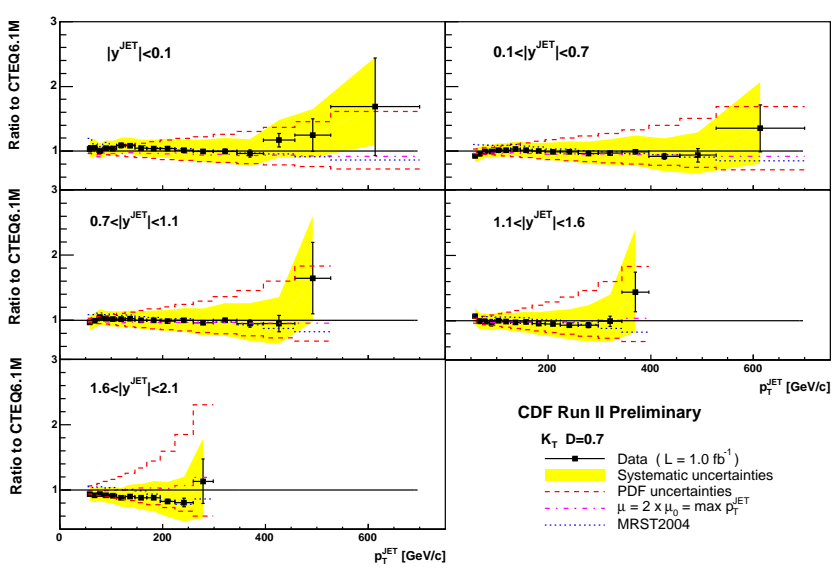

FIG. 5: Ratio of data to theory as function of $p_{T}^{\text {jet }}$ in five different rapidity regions



FIG. 6: The inclusive jet cross section with $k_{T}$ algorithm for $0.1<|Y|<0.7$ using $D=0.5$ (left) and $D=1.0$ (right).

rithm can be used at hadron-hadron colliders, once the nonperturbative corrections discussed above are performed. In this proceedings we report new results obtained with $1.0 \mathrm{fb}^{-1}$ of data and extended to forward rapidities. Fig. 4 shows the measured inclusive jet cross section with $k_{T}$ algorithm with parameter $D=0.7$ for jets with $p_{T}^{\text {jet }}>54 \mathrm{GeV} / \mathrm{c}$ in five jet rapidity regions. The different rapidity region cross sections are scaled by a given factor for presentation purposes.

Fig. 5 shows the ratio of data to theory with shaded band representing total experimental statistical (systematic) uncertainty. The theoretical predictions are obtained with NLO pQCD program JETRAD [11] with CTEQ6.1M PDF and the renormalization and factorization scales set to $\mu_{0}=$ $\max \left(p_{T}^{\text {jet }}\right) / 2$. The theoretical predictions include a correction for non-perturbative contributions. Different sources of uncertainties for theoretical predictions were considered, with the main contribution coming from the uncertainty on the PDFs and could account to as much as ${ }_{-40 \%}^{+130 \%}$ for forward jets in $1.6<|Y|<2.1$ region. The dotted lines present the ratios of theoretical predictions with MRST2004 and CTEQ6.1M PDFs as an input. The dotted-dashed lines show the ratios of predictions with $2 \mu_{0}$ and $\mu_{0}$, which changes the predictions only by few percent. Good agreement is observed between the measured cross section and the theoretical predictions.

Similar good agreement between data and theory is observed using a $D$ parameter of 0.5 and 1.0 , see Fig. 6 , demonstrating that soft contributions are well under control.

\section{CONCLUSIONS}

The CDF collaboration is carrying out very intense physics program. The amount of collected data, that currently exceeds $1.0 \mathrm{fb}^{-1}$, is bringing a new level of QCD precision studies at hadron-hadron colliders. Measurements of the inclusive jet cross section with two different jet algorithms show a good agreement with NLO pQCD calculations. The jet measurements in forward regions are placing significant constrains on gluon PDFs at high $x$, thus allowing to reduce theoretical uncertainties associated with parton distribution functions for predictions to many physics processes at the Tevatron and LHC.

\section{Acknowledgments}

I would like to thank the organizers of XXXVI International Symposium on Multiparticle Dynamics for a kind invitation, warm hospitality, and for an exciting conference.
[1] T. Affolder et al., Phys. Rev. D 64, 032001 (2001).

[2] J. Pumplin et al., JHEP 0207, 012 (2002); A.D. Martin et al., Eur. Phys. J. C 23, 73 (2002).

[3] G.C. Blazey et al., hep-ex0005012.

[4] S. Catani et al., Nucl. Phys. B 406, 187 (1993); S.D. Ellis and D.E. Soper, Phys. Rev. D 48, 3160 (1993).

[5] A. Bhatti et al., Nucl. Instrum. Meth. A 566, 375 (2006).

[6] S.D. Ellis et al., Phys. Rev. Lett. 64, 2121 (1990).
[7] T. Sjostrand et al., Comput.Phys.Commun. 135, 238 (2001).

[8] T. Affolder et al., Phys. Rev. D 65, 092002 (2002).

[9] G. Corcella et al., JHEP 0101, 010 (2001).

[10] A. Abulencia et al., Phys. Rev. Lett. 96, 122001 (2006).

[11] W.T. Giele, W.N. Glocer and D.A.Kosower, Nucl. Phys. B 403, 633 (1993). 